Louisiana State University

LSU Digital Commons

Faculty Publications

Department of Mathematics

7-1-2020

\title{
Sequential Predictors for Delay Compensation for Perturbed Discrete Time Systems
}

Frederic Mazenc

Universite Paris-Saclay

Michael Malisoff

Universite Paris-Saclay

Follow this and additional works at: https://digitalcommons.Isu.edu/mathematics_pubs

\section{Recommended Citation}

Mazenc, F., \& Malisoff, M. (2020). Sequential Predictors for Delay Compensation for Perturbed Discrete

Time Systems. Proceedings of the American Control Conference, 2020-July, 1696-1700. https://doi.org/ 10.23919/ACC45564.2020.9148032

This Conference Proceeding is brought to you for free and open access by the Department of Mathematics at LSU Digital Commons. It has been accepted for inclusion in Faculty Publications by an authorized administrator of LSU Digital Commons. For more information, please contact ir@lsu.edu. 


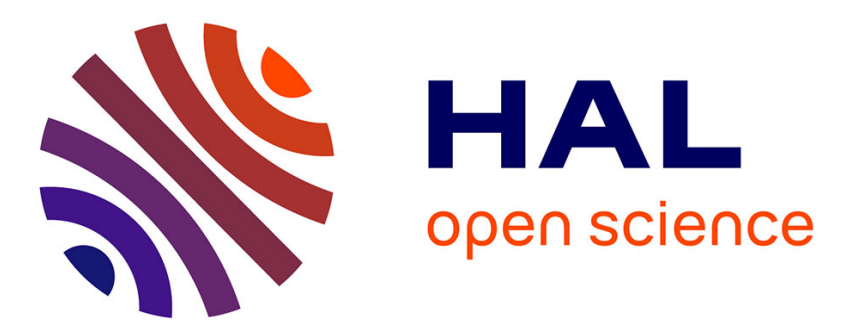

\section{Sequential Predictors for Delay Compensation for Perturbed Discrete Time Systems}

Frederic Mazenc, Michael Malisoff

\section{To cite this version:}

Frederic Mazenc, Michael Malisoff. Sequential Predictors for Delay Compensation for Perturbed Discrete Time Systems. ACC 2020 - American Control Conference, Jul 2020, Denver, United States. hal-03113797

\section{HAL Id: hal-03113797 https://hal.inria.fr/hal-03113797}

Submitted on 18 Jan 2021

HAL is a multi-disciplinary open access archive for the deposit and dissemination of scientific research documents, whether they are published or not. The documents may come from teaching and research institutions in France or abroad, or from public or private research centers.
L'archive ouverte pluridisciplinaire HAL, est destinée au dépôt et à la diffusion de documents scientifiques de niveau recherche, publiés ou non, émanant des établissements d'enseignement et de recherche français ou étrangers, des laboratoires publics ou privés. 


\section{Sequential Predictors for Delay Compensation for Perturbed Discrete Time Systems}

\author{
Frederic Mazenc
}

\begin{abstract}
We provide sequential predictors for delayed timevarying discrete time linear systems with outputs, where the system and output measurements are perturbed by uncertainty. Our predictors ensure input-to-state stability with respect to uncertainties in the system and in the output, under arbitrarily long input delays. Our strategy is to introduce a number of dynamical extensions that equals the length of the input delay. Our example illustrates the usefulness of our control design.
\end{abstract}

Index Terms-Delay, robustness, discrete time, prediction

\section{INTRODUCTION}

Delay compensation is a central topic in control theory, and is motivated by input delays in important engineering applications; see for instance [12] and [16]. The delay compensation problem usually entails designing a feedback control that can be computed from time lagged state or output measurements and which renders a system uniformly globally asymptotically stable to an equilibrium. While the delay compensation literature is largely focused on continuous time systems, there are significant applications that lead to discrete time systems; see for instance [8], [9], and [17].

One approach to input delay compensation is emulation, where no information about the input delay is used in the control design, and where one then seeks upper bounds on the delays for which the control still ensures global asymptotic stability, when the current measurements are replaced by delayed measurements in the feedback. Emulation often makes it possible to use more basic feedback control designs for undelayed systems, and involves transforming a Lyapunov function for the closed loop undelayed systems into a Lyapunov-Krasovskii functional for the corresponding delayed system [14]. However, emulation may only provide conservative estimates of the maximum delays that the system can tolerate, and so cannot always cope with delays that are long relative to the total response time of the system.

This motivated a large literature on alternative delay compensation methods, where values of the delay can be incorporated into the control design, such as [4], [20], [21] and [22]. One such method is the reduction model approach, which was developed in [2]; see also [15] for later work on time-varying systems. The reduction model approach shares the desirable feature with the prediction approaches in [12] that it can compensate for arbitrarily long input delays.

Mazenc is with EPI DISCO INRIA-Saclay, Laboratoire des Signaux et Systèmes (L2S, UMR CNRS 8506), CNRS, CentraleSupélec, Université Paris-Sud, 3 rue Joliot Curie, 91192, Gif-sur-Yvette, France, frederic.mazenc@12s.centralesupelec.fr.

Malisoff is with the Department of Mathematics, Louisiana State University, Baton Rouge, LA 70803-4918, USA, malisoff@1su.edu.

Malisoff was supported by NSF Grant 1711299 .

\author{
Michael Malisoff
}

However, a potential challenge for reduction or standard prediction approaches is that their controls usually require storing past control values over an interval of times (or, in the continuous time case, are only implicitly defined as solutions of integral equations that do not admit explicit solutions).

Sequential predictors (which were introduced in [3]) provide another delay compensation method, where the distributed terms in standard predictive controls are replaced by dynamic extensions, which contain copies of the original system running on different time scales; see [5] and [13]. By eliminating the distributed terms in the controls, sequential predictors may provide a useful alternative to coping with the computational challenges associated with standard predictive controls. However, we believe that sequential predictors had not been developed for input delay compensation in timevarying discrete time systems with output feedbacks.

Discrete time systems can model sampling in controls, and also naturally arise in modeling discrete event systems, which are prone to input delays that are analogous to the delays in continuous time systems. This motivates the present work, which provides a discrete time analog of our continuous time delay compensating work [13]. The analog covers discrete time linear time-varying systems with constant input delays, where the state dynamics and its output are perturbed by uncertainties. We provide an input-to-state stability (or ISS) result with respect to the uncertainties, while also compensating for arbitrarily long input delays without any distributed terms in the controls. This contrasts with the notable work [8] on discretization based control whose sums are analogous to the integral terms that arise for continuous time systems.

We use the following standard notation, where the dimensions of our Euclidean spaces are arbitrary unless otherwise noted. Let $|\cdot|$ denote the usual Euclidean norm and the corresponding matrix norm, and $|\cdot|_{\infty}$ be the corresponding sup norm. Let $|\cdot|_{\mathcal{I}}$ be the supremum over an interval $\mathcal{I}$. Let $\mathcal{K} \mathcal{L}$ and $\mathcal{K}_{\infty}$ be the usual classes of comparison functions from [11, Chapter 4], and $I_{n}$ be the $n$ dimensional identity matrix. A time-varying discrete time system of the form

$$
X_{k+1}=f\left(k, X_{k}, X_{k-r}, D_{k}\right)
$$

with state space $\mathbb{R}^{n}$ and a constant integer delay $r \geq 0$ is called input-to-state stable (which is also abbreviated as ISS) with respect to the sequence $D_{k} \in \mathbb{R}^{d}$ provided there are $\beta \in \mathcal{K} \mathcal{L}$ and $\gamma \in \mathcal{K}_{\infty}$ such that for each integer initial time $k_{0} \geq 0$ and each $\mathbb{R}^{n}$-valued initial function $\phi$ for (1) (with $\phi$ having domain $\left[-r+k_{0}, k_{0}\right]$ ), we have

$$
\left|X_{k}\right| \leq \beta\left(|\phi|_{\infty}, k-k_{0}\right)+\gamma\left(|D|_{\left[k_{0}, k\right]}\right)
$$


for all $k \geq k_{0}$ and all choices of the $D_{k}$ 's (which is equivalent to (2) with $|D|_{\left[k_{0}, k\right]}$ replaced by $|D|_{\infty}$, by causality). However, for our state dynamics in our theorem, we choose the initial times $k_{0}$ for the state to always be $k_{0}=0$, and we choose constant initial functions at $k_{0}=0$. For square matrices $M_{1}$ and $M_{2}$ of the same size, we use $M_{1} \leq M_{2}$ to mean that $M_{2}-M_{1}$ is nonnegative definite.

\section{MAin Result}

\section{A. Assumptions and Statement of Theorem}

We study linear time-varying systems of the form

$$
\left\{\begin{aligned}
x_{k+1} & =A_{k} x_{k}+B_{k} u_{k-r}+d_{k} \\
y_{k} & =C_{k} x_{k}+v_{k}
\end{aligned}\right.
$$

where the sequences $A_{k} \in \mathbb{R}^{n \times n}, B_{k} \in \mathbb{R}^{n \times m}, C_{k} \in \mathbb{R}^{s \times n}$ of matrices are known, but where the sequences $d_{k} \in \mathbb{R}^{n}$ and $v_{k} \in \mathbb{R}^{s}$ of vectors represent uncertainties and so are not assumed to be known. The integer $r \geq 1$ represents an input delay, and the sequence $u_{k} \in \mathbb{R}^{m}$ represents an input, which we later choose to be an output feedback control to achieve our control objective of ensuring that the closed loop system enjoys an ISS property with respect to the combined disturbance sequence $\left(d_{k}, v_{k}\right)$. We will assume the following:

Assumption 1: The sequences of known matrices $A_{k} \in$ $\mathbb{R}^{n \times n}, B_{k} \in \mathbb{R}^{n \times m}$, and $C_{k} \in \mathbb{R}^{s \times n}$ in (3) are bounded. Also, there exist known bounded sequences $K_{k} \in \mathbb{R}^{m \times n}$ and $L_{k} \in \mathbb{R}^{n \times s}$ such that the systems

$$
p_{k+1}=\left(A_{k}+B_{k} K_{k}\right) p_{k}+q_{k}
$$

and

$$
r_{k+1}=\left(A_{k}+L_{k} C_{k}\right) r_{k}+s_{k}
$$

are ISS with respect to the disturbances $q_{k}$ and $s_{k}$.

In the special case where the sequences $A_{k}, B_{k}$, and $C_{k}$ are constant matrices that we denote by $A, B$, and $C$ respectively, the preceding assumption is satisfied if $(A, B)$ is controllable and $(A, C)$ is observable; this follows by choosing constant matrices $K$ and $L$ such that $A+B K$ and $A+L C$ are Schur stable and then choosing the constant sequences $K_{k}=K$ and $L_{k}=L$, using the reasoning from [9, Example 3]. See also Section III for conditions under which the assumption is satisfied for time-varying cases, and for cases where the system (3) arises from sampled-data continuous time systems. Our main result is as follows:

Theorem 1: Let Assumption 1 hold, and choose sequences $K_{k}$ and $L_{k}$ that satisfy the requirements from Assumption 1. Consider the system (3) in closed loop with the control

$$
u_{k}=K_{k+r} z_{k}^{r}
$$

where $z_{k}^{r}$ is the state of the last $n$ components of the $\mathbb{R}^{n r}$ dimensional dynamical extension that is defined by

$$
\left\{\begin{aligned}
z_{k+1}^{1} & =A_{k+1} z_{k}^{1}+B_{k+1} u_{k-r+1}+\alpha_{1, k} \\
z_{k+1}^{2} & =A_{k+2} z_{k}^{2}+B_{k+2} u_{k-r+2}+\alpha_{2, k} \\
& \vdots \\
z_{k+1}^{r} & =A_{k+r} z_{k}^{r}+B_{k+r} u_{k}+\alpha_{r, k},
\end{aligned}\right.
$$

with the constant initial functions being defined by $z_{k}^{i}=0$ for $i=1, \ldots, r$ and all integers $k \leq 0$, and with the choices

$$
\begin{aligned}
\alpha_{1, k}= & L_{k+1} C_{k+1} z_{k}^{1}-L_{k+1} y_{k+1} \\
\alpha_{2, k}= & L_{k+2} C_{k+2}\left[z_{k}^{2}-A_{k+1} z_{k}^{1}-B_{k+1} u_{k-r+1}\right] \\
\vdots & \\
\alpha_{r, k}= & L_{k+r} C_{k+r}\left[z_{k}^{r}-A_{k+r-1} z_{k}^{r-1}\right. \\
& \left.-B_{k+r-1} u_{k-1}\right] .
\end{aligned}
$$

Then (3) in closed loop with the dynamic control given by (6), (7), and (8) is input-to-state stable with respect to the combined disturbance $\left(d_{k}, v_{k}\right)$ on its state space $\mathbb{R}^{n}$.

Remark 1: We refer to each $\mathbb{R}^{n}$-valued $z^{i}$ dynamic in (7) as a sequential predictor. The sequential predictors are interconnected with the original system in a chain, which is a reason why sequential predictors are also called chain predictors. However, only the states of the last predictor (with values $z_{k}^{r}$ ) are used in the control formula (6).

Remark 2: The sequential predictors in Theorem 1 share features with the corresponding results for the continuous time case in [13], insofar that they consist of multiple copies (7) of the original system running on different time scales, with additional stabilizing terms (8) added to each of the dynamic extensions. However, a notable difference between the continuous and discrete time sequential prediction results is in the number of dynamical extensions. For instance, in [13], the number $m$ of sequential predictors was required to satisfy $m>11.4 k h$ where $h$ was the constant delay and $k$ was a Lipschitz constant for the dynamics (which gives the condition $k \geq|A|$ in the special case of linear time-invariant systems $\dot{x}(t)=A x(t)+B u(t-h)$ ). However, Theorem 1 only needs $r$ sequential predictors. Our control is causal, because while $y_{k+1}$ appears in (8), we only use the output values $y_{k-\ell}$ with $\ell \geq 0$ to compute the control value $u_{k-r}$ at each time $k$.

\section{B. Sketch of Proof of Theorem 1}

We introduce the error variables

$$
e_{k}^{i}=z_{k}^{i}-z_{k+1}^{i-1}
$$

for all $k$ and $i \in\{1,2, \ldots, r\}$, where $z_{k}^{0}=x_{k}$ for all $k \geq 0$. We also set $\alpha_{0, k}=d_{k}$ for all $k \geq 0$. Then

$$
\begin{aligned}
e_{k+1}^{i}= & A_{k+i} z_{k}^{i}+B_{k+i} u_{k-r+i}+\alpha_{i, k}-z_{k+2}^{i-1} \\
= & A_{k+i} z_{k}^{i}+B_{k+i} u_{k-r+i}+\alpha_{i, k} \\
& -\left[A_{k+i} z_{k+1}^{i-1}+B_{k+i} u_{k-r+i}+\alpha_{i-1, k+1}\right] \\
= & A_{k+i}\left[z_{k}^{i}-z_{k+1}^{i-1}\right]+\alpha_{i, k}-\alpha_{i-1, k+1} .
\end{aligned}
$$

Here and in the sequel, all equalities and inequalities should be understood to hold for all integers $k \geq 0$ and $i \in$ $\{1,2, \ldots, r\}$, unless otherwise indicated. Therefore,

$$
\begin{aligned}
e_{k+1}^{1}= & A_{k+1}\left[z_{k}^{1}-z_{k+1}^{0}\right]+\alpha_{1, k}-\alpha_{0, k+1} \\
= & A_{k+1}\left[z_{k}^{1}-A_{k} x_{k}-B_{k} u_{k-r}\right] \\
& +\alpha_{1, k}-A_{k+1} d_{k}-d_{k+1} \text { and } \\
e_{k+1}^{i}= & A_{k+i}\left[z_{k}^{i}-A_{k+i-1} z_{k}^{i-1}\right. \\
& \left.-B_{k+i-1} u_{k-r+i-1}-\alpha_{i-1, k}\right] \\
+ & \alpha_{i, k}-\alpha_{i-1, k+1} \text { for all } i \in\{2, \ldots, r\} .
\end{aligned}
$$


Also, our choice of $\alpha_{1, k}$ from (8) gives

$$
\begin{aligned}
\alpha_{1, k}= & L_{k+1} C_{k+1}\left(z_{k}^{1}-A_{k} x_{k}-B_{k} u_{k-r}\right. \\
& \left.-d_{k}\right)-L_{k+1} v_{k+1} .
\end{aligned}
$$

From (8) and (11)-(13), it follows that

$$
\begin{aligned}
e_{k+1}^{1}= & \left(A_{k+1}+L_{k+1} C_{k+1}\right) e_{k}^{1}-L_{k+1} v_{k+1}-d_{k+1} \\
e_{k+1}^{i}= & -A_{k+i} \alpha_{i-1, k}-\alpha_{i-1, k+1} \\
& +\left(A_{k+i}+L_{k+i} C_{k+i}\right)\left(z_{k}^{i}-A_{k+i-1} z_{k}^{i-1}\right. \\
& \left.-B_{k+i-1} u_{k-r+i-1}\right) \\
= & -A_{k+i} \alpha_{i-1, k}-\alpha_{i-1, k+1} \\
& +\left(A_{k+i}+L_{k+i} C_{k+i}\right)\left(z_{k}^{i}-z_{k+1}^{i-1}+\alpha_{i-1, k}\right) \\
= & L_{k+i} C_{k+i} \alpha_{i-1, k}-\alpha_{i-1, k+1} \\
& +\left(A_{k+i}+L_{k+i} C_{k+i}\right) e_{k}^{i} \text { for } i \geq 2 .
\end{aligned}
$$

Also, (8) and (13) give

$$
\begin{aligned}
\alpha_{1, k}= & L_{k+1} C_{k+1} e_{k}^{1}-L_{k+1} v_{k+1} \text { and } \\
\alpha_{i, k}= & L_{k+i} C_{k+i}\left[z_{k}^{i}-A_{k+i-1} z_{k}^{i-1}\right. \\
& \left.-B_{k+i-1} u_{k-r+i-1}-\alpha_{i-1, k}\right] \\
& +L_{k+i} C_{k+i} \alpha_{i-1, k} \\
= & L_{k+i} C_{k+i}\left[z_{k}^{i}-z_{k+1}^{i-1}\right] \\
& +L_{k+i} C_{k+i} \alpha_{i-1, k} \\
= & L_{k+i} C_{k+i}\left[e_{k}^{i}+\alpha_{i-1, k}\right] \text { for } i \geq 2 .
\end{aligned}
$$

Since the $e^{1}$ dynamics in (14) can be written in the form

$$
r_{k+2}=\left(A_{k+1}+L_{k+1} C_{k+1}\right) r_{k+1}+s_{k+1}
$$

with

$$
r_{k}=e_{k-1}^{1} \text { and } s_{k}=-L_{k} v_{k}-d_{k},
$$

it follows from Assumption 1 that there are $\beta_{1}^{*} \in \mathcal{K} \mathcal{L}$ and $\gamma_{1}^{*} \in \mathcal{K}_{\infty}$ such that

$$
\left|r_{\ell}\right| \leq \beta_{1}^{*}\left(\left|r_{2}\right|, \ell-2\right)+\gamma_{1}^{*}\left(|(d, v)|_{\infty}\right)
$$

for all $\ell \geq 2$. Since our assumption that the initial functions for the $z_{k}^{i}$,s are 0 implies that $e_{0}^{1}=-x_{1}=-A_{0} x_{0}-d_{0}$ and

$$
\begin{aligned}
e_{1}^{1} & =z_{1}^{1}-x_{2}=z_{1}^{1}-A_{1} x_{1}-B_{1} K_{1} z_{1-r}^{r}-d_{1} \\
& =z_{1}^{1}-A_{1} x_{1}-d_{1} \\
& =-\left(A_{1}+L_{1} C_{1}\right)\left(A_{0} x_{0}+d_{0}\right)-d_{1}-L_{1} v_{1},
\end{aligned}
$$

this provides functions $\beta_{1} \in \mathcal{K} \mathcal{L}$ and $\gamma_{1} \in \mathcal{K}_{\infty}$ such that

$$
\max \left\{\left|e_{k}^{1}\right|,\left|\alpha_{1, k}\right|\right\} \leq \beta_{1}\left(\left|x_{0}\right|, k\right)+\gamma_{1}\left(|(d, v)|_{\infty}\right)
$$

for all $k \geq 0$ and initial states and disturbances $(d, v)$, by using the fact that $\gamma(a+b) \leq \gamma(2 a)+\gamma(2 b)$ holds for all functions $\gamma \in \mathcal{K}_{\infty}$ and nonnegative $a$ and $b$ and the fact that class $\mathcal{K} \mathcal{L}$ functions are of class $\mathcal{K}_{\infty}$ in their first argument.

We now argue by induction. Our induction hypothesis is that the integer $N \in\{1, \ldots, r-1\}$ is such that there exist functions $\beta_{N} \in \mathcal{K} \mathcal{L}$ and $\gamma_{N} \in \mathcal{K}_{\infty}$ such that

$$
\max \left\{\left|e_{k}^{i}\right|,\left|\alpha_{i, k}\right|\right\} \leq \beta_{N}\left(\left|x_{0}\right|, k\right)+\gamma_{N}\left(|(d, v)|_{\infty}\right)
$$

holds for all $k \geq 0$ and all $i \in\{1,2, \ldots, N\}$. The preceding condition holds for $N=1$, by the preceding argument. To show that it holds for $N+1$ if it holds for an integer $N \in$ $[1, r)$, first note that the $e_{k}^{N+1}$ dynamics from (14) can be written in the form

$$
\begin{aligned}
r_{k+N+2}= & \left(A_{k+N+1}+L_{k+N+1} C_{k+N+1}\right) r_{k+N+1} \\
& +s_{k+N+1}
\end{aligned}
$$

with the choices

$$
\begin{aligned}
& r_{k+N+1}=e_{k}^{N+1} \text { and } \\
& s_{k+N+1}=L_{k+N+1} C_{k+N+1} \alpha_{N, k}-\alpha_{N, k+1} .
\end{aligned}
$$

By the induction hypothesis, we have

$$
\begin{aligned}
& \left|s_{k+N+1}\right| \leq \\
& \left(|L C|_{\infty}+1\right)\left(\beta_{N}\left(\left|x_{0}\right|, k\right)+\gamma_{N}\left(|(d, v)|_{\infty}\right)\right)
\end{aligned}
$$

for all $k \geq 0$. Therefore, Assumption 1 gives

$$
\begin{aligned}
\left|e_{k}^{N+1}\right|= & \left|r_{k+N+1}\right| \\
\leq & \beta_{1}^{*}\left(\left|r_{N+1}\right|, k\right) \\
& +\gamma_{1}^{*}\left(\left(|L C|_{\infty}+1\right) \beta_{N}\left(\left|x_{0}\right|, k\right)\right. \\
& \left.+\gamma_{N}\left(|(d, v)|_{\infty}\right)\right) \\
\leq & \beta_{1}^{*}\left(\left|e_{0}^{N+1}\right|, k\right)+\gamma_{1}^{*}\left(2 \gamma_{N}\left(|(d, v)|_{\infty}\right)\right) \\
& +\gamma_{1}^{*}\left(2\left(|L C|_{\infty}+1\right) \beta_{N}\left(\left|x_{0}\right|, k\right)\right)
\end{aligned}
$$

for all $k \geq 0$.

On the other hand, our assumptions that the initial functions for the $z_{k}^{i}$,s are zero and the fact that $N<r$ give

$$
\begin{aligned}
e_{0}^{N+1}= & z_{0}^{N+1}-z_{1}^{N} \\
= & -A_{N} z_{0}^{N}-B_{N} K_{N} z_{0}^{r} \\
& -\alpha_{N, 0} \\
= & -\alpha_{N, 0}
\end{aligned}
$$

so our inductive hypotheses and the fact that class $\mathcal{K} \mathcal{L}$ functions are of class $\mathcal{K}_{\infty}$ in their first arguments give

$$
\begin{aligned}
\beta_{1}^{*}\left(\left|e_{0}^{N+1}\right|, k\right) \leq & \beta_{1}^{*}\left(2 \beta_{N}\left(\left|x_{0}\right|, 0\right), k\right) \\
& +\beta_{1}^{*}\left(2 \gamma_{N}\left(|(d, v)|_{\infty}\right), 0\right)
\end{aligned}
$$

for all $k \geq 0$, where we also used the fact that class $\mathcal{K} \mathcal{L}$ functions are nonincreasing in their second arguments. If we now combine (25) and (27), then we can find functions $\beta_{N+1}^{b} \in \mathcal{K} \mathcal{L}$ and $\gamma_{N+1}^{b} \in \mathcal{K}_{\infty}$ such that

$$
\left|e_{k}^{N+1}\right| \leq \beta_{N+1}^{b}\left(\left|x_{0}\right|, k\right)+\gamma_{N+1}^{b}\left(|(d, v)|_{\infty}\right)
$$

holds for all $k \geq 0$ and initial states and disturbances $(d, v)$. It follows from the formula for $\alpha_{N+1, k}$ (which is obtained by setting $i=N+1$ in (15)) and our inductive hypotheses that we can find functions $\beta_{N+1}^{a} \in \mathcal{K} \mathcal{L}$ and $\gamma_{N+1}^{a} \in \mathcal{K}_{\infty}$ such that

$$
\begin{aligned}
& \max \left\{\left|e_{k}^{N+1}\right|,\left|\alpha_{N+1, k}\right|\right\} \leq \\
& \beta_{N+1}^{a}\left(\left|x_{0}\right|, k\right)+\gamma_{N+1}^{a}\left(|(d, v)|_{\infty}\right) \text { for all } k \geq 0,
\end{aligned}
$$

for all initial states and $k \geq 0$. Therefore, we can choose $\beta_{N+1}=\max \left\{\beta_{N+1}^{a}, \beta_{N}\right\}$ and $\gamma_{N+1}=\max \left\{\gamma_{N+1}^{a}, \gamma_{N}\right\}$.

Choosing $N=r-1$ in the preceding argument, we get

$$
\left|e_{k}^{i}\right| \leq \beta_{r}\left(\left|x_{0}\right|, k\right)+\gamma_{r}\left(|(d, v)|_{\infty}\right)
$$

for all $k \geq 0$ and $i \in\{1, \ldots, r\}$. Recalling our assumption that the initial functions are constant at the initial time 0 (with the initial functions for the $z_{i}$ 's being zero for $i \in$ 
$\{1,2, \ldots, r\}$, we can use a telescoping sum to obtain

$$
\begin{aligned}
\left|z_{k-r}^{r}-x_{k}\right| & =\left|\sum_{i=0}^{r-1}\left(z_{k-r+i}^{r-i}-z_{k-r+i+1}^{r-i-1}\right)\right| \\
& \leq \sum_{i=0}^{r-1}\left|e_{k-r+i}^{r-i}\right| \\
& \leq r \beta_{r}\left(\left|x_{0}\right|, k\right)+r \gamma_{r}\left(|(d, v)|_{\infty}\right)
\end{aligned}
$$

for all $k \geq r$, where the first inequality used the triangle inequality. Therefore, we can write the closed loop system from the statement of our theorem in the form

$$
\begin{aligned}
x_{k+1} & =A_{k} x_{k}+B_{k} K_{k} z_{k-r}^{r}+d_{k} \\
& =\left(A_{k}+B_{k} K_{k}\right) x_{k}+q_{k}
\end{aligned}
$$

where the sequence $q_{k}=B_{k} K_{k}\left(z_{k-r}^{r}-x_{k}\right)+d_{k}$ satisfies

$$
\left|q_{k}\right| \leq \bar{b}\left(r \beta_{r}\left(\left|x_{0}\right|, k\right)+r \gamma_{r}\left(|(d, v)|_{\infty}\right)\right)+|d|_{\infty}
$$

for all $k \geq r$, where $\bar{b}=|B K|_{\infty}$.

Also, the linear growth of the dynamics provides a function $\underline{\gamma} \in \mathcal{K}_{\infty}$ such that $\max \left\{\left|x_{k}\right|,\left|z_{k}^{i}\right|\right\} \leq \underline{\gamma}\left(\left|x_{0}\right|\right)+$ $\gamma\left(|(\overline{d, v})|_{\infty}\right)$ for all integers $k \in[0, r]$ and $i=1,2, \ldots r$, and therefore also a $\underline{\gamma}_{a} \in \mathcal{K}_{\infty}$ such that $\left|q_{k}\right| \leq \underline{\gamma}_{a}\left(\left|x_{0}\right|\right) \max \{r-$ $\left.k+1,0\}+\left.\underline{\gamma}_{a}(\overline{\mid(} d, v)\right|_{\infty}\right)$ for all $k \in[0, \bar{r}]$, which we can combine with (33) to find functions $\beta_{b} \in \mathcal{K} \mathcal{L}$ and $\gamma_{b} \in \mathcal{K}_{\infty}$ such that $\left|q_{k}\right| \leq \beta_{b}\left(\left|x_{0}\right|, k\right)+\gamma_{b}\left(|(d, v)|_{\infty}\right)$ for all $k \geq 0$. Hence, Assumption 1 provides functions $\beta_{1} \in \mathcal{K} \mathcal{L}$ and $\gamma_{1} \in \mathcal{K}_{\infty}$ such that

$$
\begin{aligned}
\left|x_{k}\right| \leq & \beta_{1}\left(\left|x_{f(k / 2)}\right|, k-f(k / 2)\right) \\
& +\gamma_{1}\left(\beta_{b}\left(\left|x_{0}\right|, f(k / 2)\right)+\gamma_{b}\left(|(d, v)|_{\infty}\right)\right)
\end{aligned}
$$

for all $k \geq 0$, where $f$ denotes the floor function. Also,

$$
\begin{aligned}
\left|x_{f(k / 2)}\right| \leq & \beta_{1}\left(\left|x_{0}\right|, 0\right) \\
& +\gamma_{1}\left(\beta_{b}\left(\left|x_{0}\right|, 0\right)+\gamma_{b}\left(|(d, v)|_{\infty}\right)\right) \\
\leq & \gamma_{c}\left(\left|x_{0}\right|\right)+\gamma_{c}\left(|(d, v)|_{\infty}\right),
\end{aligned}
$$

where $\gamma_{c}(s)=\beta_{1}(s, 0)+\gamma_{1}\left(2 \beta_{b}(s, 0)\right)+\gamma_{1}\left(2 \gamma_{b}(s)\right)$. The final ISS bound follows by using (35) to upper bound the $\left|x_{f(k / 2)}\right|$ in (34) and then using the subadditivity property of $\mathcal{K}_{\infty}$ functions as before.

\section{Checking Assumption 1}

We believe that the preceding result is novel, even in the special case where the coefficient matrices are constant. In this section, we provide two methods for checking Assumption 1, to illustrate the applicability of our theorem. First, we show how to apply our approach to sampled data timeinvariant continuous time systems. Then, we explain how to check Assumption 1 for cases with time-varying coefficients.

\section{A. Sampling in Continuous Time Systems}

While stated for discrete time systems, we can combine our theorem with the Kalman-Ho-Narendra criterion from [19] to compensate for arbitrarily long input delays and arbitrarily large sampling intervals, when the method is applied to continuous time data sampled systems with periodic sampling; see [1], [7], and [18] for motivation for sampling. To see how, assume that we are given a time invariant linear continuous time system of the form $\dot{x}=A_{0} x+B_{0} u$ with the control $u$ to be specified and an output $y=C_{0} x$, where we assume that $\left(A_{0}, B_{0}\right)$ is controllable and that $\left(A_{0}, C_{0}\right)$ is observable. Further assume that the constant $\delta>0$ is such that the following condition holds for every pair $(\lambda, \mu)$ of eigenvalues of $A_{0}$ and so also for every pair $(\lambda, \mu)$ of eigenvalues of $A_{0}^{\top}$ : the constant $\delta(\lambda-\mu)$ is not a nonzero integer multiple of $2 \pi i$.

Next, assume that the control $u$ is constrained to be constant on each interval of the form $[j \delta,(j+1) \delta)$ for each integer $j \geq 0$, and that the measurements $y=C_{0} x$ are only available at the discrete times $j \delta$ for integers $j \geq 0$. Then for each solution of the system, we can apply variation of parameters to obtain $x(\delta)=A x(0)+B u(0)$, where

$$
A=e^{\delta A_{0}} \text { and } B=\int_{0}^{\delta} e^{(\delta-s) A_{0}} B_{0} \mathrm{~d} s .
$$

This produces a discrete time system that is covered by our theorem, because the preceding assumptions ensure that $(A, B)$ is controllable and that $\left(A, C_{0}\right)$ is observable. This follows by applying Theorem 4 and Lemma 3.4.1 from [19, Section 3.4] to the controllable pairs $(A, B)$ and $\left(A^{\top}, C_{0}^{\top}\right)$. Then we can choose constant sequences $K$ and $L$ of matrices so that $A+B K$ and $A+L C_{0}$ are both Schur stable, which then satisfy the requirements from Assumption 1. This allows us to use Theorem 1 to compensate for arbitrarily infrequent but periodic sampling in the control and the output, which correspond to allowing arbitrarily large $\delta$ values. Moreover, the number of sequential predictors is independent of $\delta$.

\section{B. Time-Varying Coefficients}

When the bounded sequences $A_{k}, B_{k}$, and $C_{k}$ are expressed in the form $A_{k}=A_{*}+\Delta_{A, k}, B_{k}=B_{*}+\Delta_{B, k}$, and $C_{k}=C_{*}+\Delta_{C, k}$ respectively, where $\left(A_{*}, B_{*}\right)$ is controllable and $\left(A_{*}, C_{*}\right)$ is observable, then we can find bounds on the suprema $\left|\Delta_{A}\right|_{\infty}=\sup \left\{\left|\Delta_{A, k}\right|: k \geq 0\right\},\left|\Delta_{B}\right|_{\infty}=$ $\sup \left\{\left|\Delta_{B, k}\right|: k \geq 0\right\}$, and $\left|\Delta_{C}\right|_{\infty}=\sup \left\{\left|\Delta_{C, k}\right|: k \geq 0\right\}$ of their time-varying parts that ensure that Assumption 1 is satisfied. For instance, choose constant matrices $K_{*}$ and $L_{*}$ such that the matrices $M_{1}=A_{*}+B_{*} K_{*}$ and $M_{2}=$ $A_{*}+L_{*} C_{*}$ are Schur stable. Then well known converse Lyapunov theory for discrete time systems (e.g., from [10]) provides constant positive definite matrices $P_{1}$ and $P_{2}$ (which are symmetric) such that $M_{i}^{\top} P_{i} M_{i}-P_{i}=-I_{n}$ for $i=1,2$. Then, with the choices $N_{1, k}=A_{k}+B_{k} K_{*}$ and $N_{2, k}=$ $A_{k}+L_{*} C_{k}$, we can find a constant $\epsilon_{0}>0$ such that

$$
N_{i, k}^{\top} P_{i} N_{i, k}-P_{i} \leq-\epsilon_{0} I_{n}
$$

holds for $i=1,2$ and all integers $k \geq 0$, provided

$$
\begin{aligned}
& \left|\Delta_{a}^{\top} P_{1}\left[\Delta_{a}+2\left(A_{*}+B_{*} K_{*}\right)\right]\right|_{\infty}<1 \text { and } \\
& \left|\Delta_{b}^{\top} P_{2}\left[\Delta_{b}+2\left(A_{*}+L_{*} C_{*}\right)\right]\right|_{\infty}<1,
\end{aligned}
$$

where $\Delta_{a}=\Delta_{A}+\Delta_{B} K_{*}$ and $\Delta_{b}=\Delta_{A}+L_{*} \Delta_{C}$. Then we have the time invariant discrete time Lyapunov functions $V_{1}(x)=x^{\top} P_{1} x$ and $V_{2}(x)=x^{\top} P_{2} x$ for the time-varying linear systems $x_{k+1}=\left(A_{k}+B_{k} K_{*}\right) x_{k}$ and $x_{k+1}=\left(A_{k}+L_{*} C_{k}\right) x_{k}$ respectively, which are also ISS Lyapunov functions for the systems in Assumption 1 with the constant sequences $K_{k}=K_{*}$ and $L_{k}=L_{*}$. Then well- 
known ISS results for discrete time systems (e.g., from [9]) imply that Assumption 1 is satisfied. We next illustrate this point in our example.

\section{ILLUSTRATION}

We study a generalization of the example from $[6$, Section V], which differs from the example in [6] because here we allow one of the coefficient matrices to be time-varying and because we allow uncertainties. The dynamics have the form

$$
\begin{aligned}
x_{1, k+1} & =\frac{5}{4} x_{1, k}+x_{2, k}+\frac{1}{4} u_{1, k-r}+d_{1, k} \\
x_{2, k+1} & =-\frac{3}{8} x_{1, k}+\frac{1}{8} u_{2, k-r}+d_{2, k} \\
y_{k} & =C_{k} x_{k}+v_{k}
\end{aligned}
$$

where $C_{k}=C_{*}+\Delta_{C, k}, C_{*}=\left[\begin{array}{ll}1 & 0\end{array}\right]$, and $\Delta_{C, k}=\left[\begin{array}{ll}\delta_{k} & 0\end{array}\right]$ for a known function $\delta_{k}$ of $k$. The work [6] studied the case where $\delta_{k}=0, d_{1, k}=d_{2, k}=\frac{1}{9} \sin (k)$, and $v_{k}=$ $\frac{1}{9} \sin \left(k^{2}\right)$ for all $k$, and it proposed an observer design, which converged to the true state value after a prescribed finite time when the perturbations $d_{i, k}$ and $v_{k}$ were replaced by zero. By contrast, here we apply Theorem 1 to (39) to build a delay compensating sequential predictor control, by applying the method from Section III-B above with the constant choices

$$
\begin{aligned}
& A_{*}=\left[\begin{array}{rr}
\frac{5}{4} & 1 \\
-\frac{3}{8} & 0
\end{array}\right], \Delta_{A, k}=0, K_{*}=\left[\begin{array}{cc}
-5 & -4 \\
3 & 0
\end{array}\right], \\
& B_{*}=\left[\begin{array}{ll}
\frac{1}{4} & 0 \\
0 & \frac{1}{8}
\end{array}\right], \Delta_{B, k}=0, \text { and } L_{*}=\left[\begin{array}{r}
-\frac{5}{4} \\
\frac{3}{8}
\end{array}\right],
\end{aligned}
$$

but similar reasoning applies for time-varying coefficients in the system, by choosing nonzero $\Delta_{A, k}$ and $\Delta_{B, k}$ values. Then our requirements from Section III-B are satisfied using the positive definite (symmetric) matrices

$$
P_{1}=I_{2} \text { and } P_{2}=\left[\begin{array}{ll}
1 & 0 \\
0 & 2
\end{array}\right] \text {. }
$$

Then (38) becomes the condition $\mid \Delta_{C}^{\top} L_{*}^{\top} P_{2}\left(L_{*} \Delta_{C}+2\left(A_{*}+\right.\right.$ $\left.\left.L_{*} C_{*}\right)\right)\left.\right|_{\infty}<1$, which can be written as

$$
\sup _{k \geq 0}\left|\left[\begin{array}{cc}
\frac{59}{32} \delta_{k}^{2} & -\frac{5}{2} \delta_{k} \\
0 & 0
\end{array}\right]\right|<1 .
$$

This is equivalent to $\sup _{k \geq 0}\left|\delta_{k}\right| \sqrt{3.399 \delta_{k}^{2}+6.25}<1$. Then the argument from Section III-B implies that we can compensate for any constant integer delay $r>1$, using the $r$ sequential predictors that are defined in Theorem 1.

\section{CONCLUSIONS}

We provided a new delay compensation method for timevarying discrete time linear systems with outputs, which made it possible to compensate for arbitrarily long input delays. Our sequential predictor approach was based on introducing a number of dynamical extensions equaling the length of the input delay. By proving input-to-state stability, we quantified the effects of uncertainties in the system dynamics and in the output. Our discrete time systems can model the effects of sampled inputs and sampled outputs in continuous time systems, in which case we can allow arbitrarily long sampling intervals in the periodic sampling. We hope to extend our results to allow time-varying delays, where there can be different delays in the measurement and in the input, and to merge our continuous and discrete time results to cover hybrid systems with input and measurement delays.

\section{REFERENCES}

[1] V. Andrieu and M. Nadri. Observer design for Lipschitz systems with discrete time measurements. In Proceedings of the 49th IEEE Conference on Decision and Control, pages 6522-6527, Atlanta, GA, 2010.

[2] Z. Artstein. Linear systems with delayed controls: A reduction. IEEE Transactions on Automatic Control, 47(4):869-879, 1982.

[3] G. Besancon, D. Georges, and Z. Benayache. Asymptotic state prediction for continuous-time systems with delayed input and application to control. In Proceedings of the European Control Conference, pages 1786-1791, Kos, Greece, 2007.

[4] F. Cacace, F. Conte, A. Germani, and P. Pepe. Stabilization of strict-feedback nonlinear systems with input delay using closed-loop predictors. International Journal of Robust and Nonlinear Control, 26(16):3524-3540, 2016.

[5] F. Cacace and A. Germani. Output feedback control of linear systems with input, state and output delays by chains of predictors. Automatica, 85:455-461, 2017.

[6] T. Dinh, F. Mazenc, and T. Raissi. Finite-time guaranteed state estimation for discrete-time systems with disturbances. In Proceedings of the 4th Conference on Control and Fault-Tolerant Systems, Casablanca, Morocco, 2019.

[7] E. Fridman. A refined input delay approach to sampled-data control. Automatica, 46:421-427, 2010.

[8] P. Garcia, A. Gonzalez, P. Castillo, R. Lozano, and P. Albertos. Robustness of a discrete-time predictor-based controller for timevarying measurement delay. IFAC Proceedings Volumes, 43(2):367$372,2010$.

[9] Z-P. Jiang and Y. Wang. Input-to-state stability for discrete-time nonlinear systems. Automatica, 37:857-869, 2001.

[10] R. Kalman and J. Bertram. Control system analysis and design via the second method of Lyapunov, Part II, discrete-time systems. Journal of Basic Engineering, 82(2):394-400, 1960.

[11] H. Khalil. Nonlinear Systems, Third Edition. Prentice Hall, Upper Saddle River, NJ, 2002.

[12] M. Krstic. Delay Compensation for Nonlinear, Adaptive, and PDE Systems. Birkhauser, Boston, 2009.

[13] F. Mazenc and M. Malisoff. Stabilization of nonlinear time-varying systems through a new prediction based approach. IEEE Transactions on Automatic Control, 62(6):2908-2915, 2017.

[14] F. Mazenc, M. Malisoff, and Z. Lin. Further results on input-to-state stability for nonlinear systems with delayed feedbacks. Automatica, 44:2415-2421, 2008.

[15] F. Mazenc, M. Malisoff, and S-I. Niculescu. Reduction model approach for linear time-varying systems with delays. IEEE Transactions on Automatic Control, 59(8):2068-2081, 2014.

[16] S. Mondie and W. Michiels. Finite spectrum assignment of unstable time delay system with a safe implementation. IEEE Transactions on Automatic Control, 48(12):2207-2212, 2003.

[17] M. Nadri, H. Hammouri, and C. Astorga. Observer design for continuous-discrete time state affine systems up to output injection. European Journal of Control, 10(3):252-263, 2004.

[18] M. Nadri, H. Hammouri, and R. Mota-Grajales. Observer design for uniformly observable systems with sampled measurements. IEEE Transactions on Automatic Control, 58(3):757-762, 2013.

[19] E. Sontag. Mathematical Control Theory, Second Edition. Springer, New York, 1998.

[20] B. Zhou. Pseudo-predictor feedback stabilization of linear systems with time-varying input delays. Automatica, 50:2861-2871, 2014.

[21] B. Zhou. Truncated Predictor Feedback for Time-Delay Systems. Springer-Verlag, Berlin, Germany, 2014.

[22] B. Zhou, Z. Lin, and G-R. Duan. Truncated predictor feedback for linear systems with long time-varying input delays. Automatica, 48:2387-2399, 2012. 